Originales

\title{
Incremento notable del cáncer de pulmón en mujeres españolas desde el año 2000
}

M. Bernal, G. Gómez, F. Gómez y Comité de Tumores del Tórax del H.C.U., de Zaragoza

\section{Resumen}

- Propósito: Con el objeto de comprobar si está incrementando el cáncer de pulmón en mujeres de ha realizado un estudio epidemiológico.

- Material y método: Se han utilizado los casos nuevos de cáncer de pulmón en mujeres que se han recogido en un registro de cáncer. Se han seguido 150.000 mujeres año, durante 15 años.

- Resultados: El cáncer de pulmón ha pasado de una tasa de incidencia de 3,2 casos *100.000 mujeres a 15,12 durante el periodo estudiado con una pendiente de 0,94 ( $\mathrm{p}<0,001)$.

- Conclusiones: Entre 1992 y 2004 el cáncer de pulmón en mujeres aumentó significativamente.

\section{Palabras clave:}

Incidencia. Cáncer de pulmón. Mujeres.

Oncología, 2005; 28 (6):282-287 


\section{Summary}

- Purpose: To determine the lung cancer incidence in Spanish women during the year 2004, and to compare it with the incidences reported in previous studies.

- Material and methods: 150,000 women were followed up during 15 years, and the diagnosis of lung cancer evaluated from a cancer registry.

- Results: The crude incidence rate was 3.3 cases per 100,000 inhabitants in 1992 and 15.12 in 2004, corresponding to a slope of $0.94(\mathrm{p}<0.001)$.

- Conclusions: The lung cancer incidence of the Spanish women between 1992 and 2005 showed a significant increase.

Key words: Lung cancer. Incidence. Women.

\section{Introducción}

En el año 2000 comunicamos el primer incremento de los casos de cáncer de pulmón detectados en las mujeres españolas ${ }^{1}$. Hasta entonces, existía una diferencia notable y estadísticamente significativa entre los casos de cáncer de pulmón en mujeres del norte de Europa y de EEUU respecto a España en el sentido de las bajas tasas de incidencia de estas. Podíamos intuir que tenia que producirse la igualdad en este aspecto y los primeros indicios se manifestaron en el año 2000.

Posteriormente hemos seguido la tendencia de estos casos para comprobar si realmente se trataba de un hallazgo real u ocasional. En aquel momento la tendencia presentaba una pendiente de $0,62(\mathrm{p}=0,0002)$.

El objetivo de este trabajo ha sido el de conocer la epidemiología del cáncer de pulmón en las mujeres de Zaragoza en el año 2004 y comprobar si el ligero incremento que se observó en el año 2000 había seguido de una manera consistente.

\section{Material y Método}

Como material se ha utilizado los casos nuevos de cáncer que se han recogido en un registro de base poblacional realizado a partir de uno hospitalario.

Se trata de un registro poblacional del Área Sanitaria III de la provincia de Zaragoza que abarca, en el año 2004, 300.000 habitantes distribuidos en zona rural y zona urbana. El número total de mujeres que se han seguido ha sido de 150.000 mujeres año, en un seguimiento desde 1990 hasta 2004. Por tanto, el número total de mujeres que se han controlado han sido de 2.250.0000. El centro de referencia de esta zona es el Hospital Clínico Universitario de Zaragoza y tiene como hospital comarcal el de Calatayud.

Para la selección de los casos se ha comprobado que todos los canceres de pulmón de mujeres detectados sean de este Área excluyéndose los que no corresponden a la misma.

Los casos se han comprobado por diagnóstico anatomopatológico en un $95 \%$ de los casos por lo que se ha podido también expresar el tipo morfológico de cáncer y el estadio anatomopatológico. Debido a que este análisis debe de realizarse en uno de estos dos hospitales, nos permite medir los cambios de incidencia de cáncer objetivamente en un corto periodo de tiempo.

A partir de los censos correspondientes y del hallazgo de "caso nuevo" se ha calculado la incidencia anual de cáncer de pulmón en mujeres durante cada uno de los años estudiados calculándose las tasas especificas y ajustadas anuales, así como intervalos de confiaza (95\% I.C.).

Se ha calculado la media, mediana y moda de la edad de comienzo del cáncer de pulmón, así como el estadio anatomopatológico ${ }^{2}$ en el que debutan, el tipo histológico y la supervivencia por estadios.

Además se describe las pruebas diagnosticas más frecuentes y de mayor valor para el diagnostico de los tumores.

Para el cálculo de la supervivencia se utilizó la prueba de Kaplan y Meier calculando la supervivencia acumulada a 5 años. 


\section{Resultados}

La media de edad fue: 61,16; mediana: 64, moda: 45; mínimo: 22 y máximo 85.

Por estado civil: 10,16 solteras, 67,75 casadas, 15,25 Viudas, 6,7 Otras.

Porcentajes de estadio anatomopatológico del tumor al momento del diagnostico fueron en orden a frecuencia: Estadio IV: 51,1\%, estadio IIIb: 11,1\%; estadio IIIa: $7 \%$; estadio IIa $8,9 \%$; estadio IIb $4,4 \%$; estadio Ib: $2,2 \%$; estadio Ia: $13,3 \%$.

La supervivencia acumulada a cinco años fue la siguiente: Estadio IV: $1 \%$, estadio iiia: $6 \%$, IIIb: $4 \%$, IIa: 30\%; IIb: 27\%; Ia: 66\%, Ib: 40\%.

Por tipos anatomopatológicos más frecuentes fueron: Carcinoma SAI 7,63\%, Celulas grandes: 1,52\%, Carcinoma indiferenciado: 4,58\% Células pequeñas: $10,68 \%$ Carcinoma de células escamosas: 23,66\% ; Adenocarcinoma: 43,51\%; Tumor carcinoide 2,29\%; Mucoepidermoide: 0,76\%; Adenocarcinoma mucinoso: 2,29\%; Carcinoma adenoescamoso: $0,76 \%$; Leiomiosarcoma: $0,76 \%$; Blastoma pulmonar: $0,76 \%$; Tumor de células granulares: $0,76 \%$.

La extensión del tumor en el momento del diagnostico fue: Local: $32,83 \%$ de los casos, regional: 25,37\%; a distancia: $41,79 \%$.

Las investigaciones relativas al diagnostica del tumor, según el criterio de la OMS en registros de cáncer fueron:

a) Clinica+Laboratorio+diagnostico por imagen+endoscopio+histologia de tumor primario $\div$ $72,88 \%$ de los enfermos b) Clinica+Laboratorio+diagnostico por imagen+ histologia de tumor primario: $8,47 \%$ de los enfermos

c) Clinica+diagnostico por imagen+ histologia de tumor primario, en $3,38 \%$ de los pacientes

d) Clinica+Laboratorio+diagnostico por imagen+cirugía exploratoria+histologia de tumor primario, en 3,38 de los enfermos

e) La combinación de otros diagnósticos se dio en porcentajes de $1,69 \%$.

Todos estos procedimientos para el diagnóstico se demoraron por termino medio 30 días desde la primera vez que la paciente fue al hospital.

Información de mayor valor para el diagnostico del tumor fue:

$5,08 \%$ de las veces fue solamente un diagnostico por imagen, $1,09 \%$ el diagnostico se hizo por citología y/o hematológica, el 5,08\% de las veces se llego al diagnostico por anatomía patológica de las metástasis y en $88,13 \%$ de las veces se hizo el diagnostico por histología del tumor primario.

Las metástasis más frecuentes fueron :

$34,14 \%$ en ganglios linfáticos remotos, $19,51 \%$ en hueso, $9,75 \%$ en hígado, 9,75\% en pulmón, $4,87 \%$ en cerebro, $9,75 \%$ en ovario, y $2,4 \%$ en piel y diferentes órganos conjuntamente.

El lugar de residencia fue en: $42,87 \%$ en medio rural y $57,14 \%$ en medio urbano.

Las tasas de incidencia se incrementan con los años, pasando de 3,2 casos por 100.000 habitantes en 1992, a 15,2 casos por 100.000 habitantes en 2004 (Tabla I) encontrándose a lo largo del perio-

TABLA I

Tasas de incidencia de cáncer de pulmón en mujeres x 100.000

\begin{tabular}{|lcccc|}
\hline Año & T. Incidencia & $95 \%$ L.C. & T.Ajustada & I.C. \\
\hline 1992 & 3,2 & $1,01-7,32$ & 1,57 & 1,41 \\
1993 & 4,4 & $1,75-9,01$ & 2,60 & 0,95 \\
1994 & 3,74 & $1,3-8,5$ & 2,51 & 2,25 \\
1995 & $0,68-6,38$ & 1,50 & 1,52 \\
1996 & 2,49 & $3,03-11,35$ & 3,75 & 2,56 \\
1997 & 6,31 & $1,35-9,22$ & 2,95 & 2,10 \\
1998 & 4,54 & $2,16-10,02$ & 3,76 & 2,74 \\
1999 & 5,05 & $5,98-16,8$ & 6,45 & 3,58 \\
2000 & 10,7 & $6,02-16,9$ & 7,12 & 3,60 \\
2001 & 11,2 & $5,4-9,86$ & 5,79 & 2,74 \\
2002 & 8,07 & $7,01-17,9$ & 10,14 & 3,45 \\
2003 & 13,23 & $8,56-21,6$ & 11,72 & 3,95 \\
2004 & 15,49 & $8,26-21,59$ & 11,65 & 3,84 \\
\hline
\end{tabular}




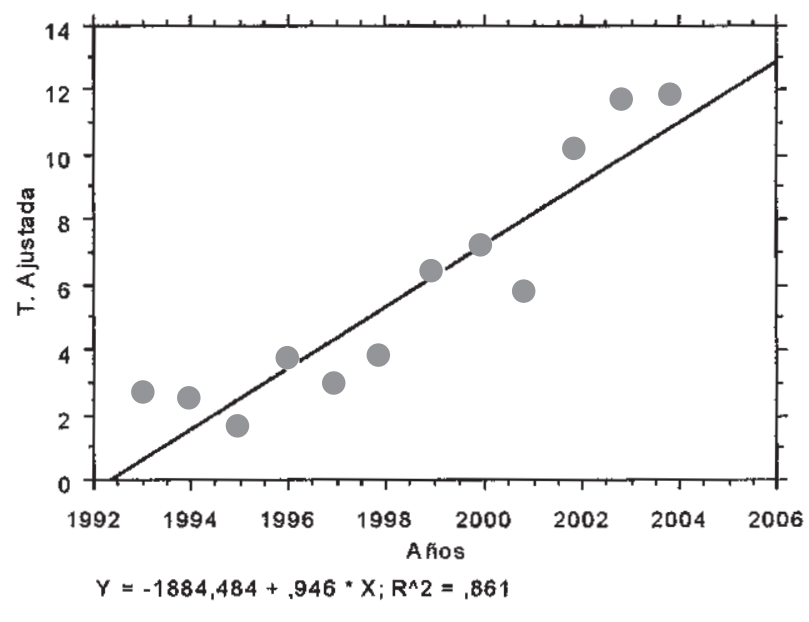

Figura 1. Tendencia de cáncer de pulmón en mujeres. Regresión lineal.

do estudiado una pendiente de 0,94 ( $\mathrm{p}<0,001)(\mathrm{Fi}-$ gura 1).

La letalidad fue de $38 \%$ y la mortalidad de 9,2 por 100.000 (4,2 ajustada).

\section{Discusión}

Como ya se apuntaba en los resultados del año 2000, las tasas de incidencia de cáncer de pulmón en mujeres y en Aragón se han ido incrementando continuando esta tendencia ascendente. Esta tendencia que ha estado liderando durante mucho tiempo en otras partes del mundo desarrollado no se había manifestado hasta la actualidad en España, a pesar de que en estos países la primera causa de morbilidad por cáncer en mujeres era el cáncer de pulmón y en España todavía continúa siendo el cáncer de mama.

Este incremento de tasas de incidencia de cáncer de pulmón en mujeres, se ha publicado también en otras partes de España a partir del año $2000^{3}$ por lo que seria plausible pensar que tales incrementos se están registrando en toda la península. Estos autores encuentran tasas de 15,93 por 100.000 habitantes, similares a las actuales que nosotros encontramos.

También en el registro de cáncer de Tarragona ${ }^{4}$ encuentran un incremento anual de cáncer de pulmón en mujeres de un 4,5\% de crecimiento anual.

En Navarra apuntan esta tendencia ${ }^{5}$ que era llamativamente descendente en otras comunidades en el comienzo de la década de los $90^{6}$.
En varios estudios de incidencia de cáncer, en Italia, muestra igualmente el incremento de cáncer de pulmón entre mujeres, en tanto que se ha visto que esta disminuyendo entre los hombres ${ }^{7-9}$.

En Holanda, en un estudio realizado entre 1989 y 1998 encuentran incrementos del cáncer de pulmón en mujeres en tanto que desciende dicho cáncer entre los hombres ${ }^{10}$.

En otro estudio realizado en Francia, en Lyón en el año 2000, con varios hospitales señalan como también es el cáncer de pulmón en mujeres uno de los que esta sufriendo un mayor incremento ${ }^{11}$.

En Inglaterra, que ha sido uno de los sitios en los que hubo mayores tasas de incidencia de cáncer de pulmón en hombres, se notifica actualmente que esta decreciendo, pero todavía incrementa algo en mujeres ${ }^{12}$.

Este marcado incremento de cáncer de pulmón en mujeres en toda Europa ${ }^{13-15}$ sugiere que como primariamente fueron los varones quienes sufrieron mayor impacto de cáncer de pulmón, también fueron quienes más tempranamente han dejado de fumar en mayor numero que las mujeres quienes, como es conocido y actualmente demostrado, se han incorporado posteriormente al habito y sufren ahora las consecuencias.

Tendencias similares ocurrieron en EEUU, y actualmente se observa un declinar del cáncer de pulmón en hombres pero todavía no ocurre en las mujeres $^{16-19}$.

La supervivencia encontrada ha sido análoga a la descrita por otros autores ${ }^{20,21}$ excepto para el estadio I que encontramos valores ligeramente superiores y para el estadio III que tenemos valores ligeramente inferiores. Nosotros pensamos que tales mínimas diferencias pueden ser debidas a que solamente estudiamos mujeres y su mediana de edad es de 64 años, además de que el principal tipo histopatológico es adenocarcinoma,

Respecto a la edad, la media de edad encontrada por Grivaux ${ }^{22}$ en el año 2000 en 137 hospitales franceses fue de 63,9 coincidiendo con la encontrada en los nuestros cuya media se sitúa en 61,16 años y la mediana: 64 .

Aunque el mayor porcentaje de tipo anatomopatológico es el adenocarcinoma, como ocurre con las estadísticas mundiales de cáncer en mujeres ${ }^{23-26}$ llama también la atención el elevado porcentaje de canceres de pulmón de células escamosas y peque- 
ñas que en su total representaría un $34 \%$ lo que explica la relación con el habito de fumar en las mujeres.

En Hong Kong, la prevalencia de hombres fumadores ha pasado de 39,7 a $22(1982-2000)^{27}$ y en mujeres de 5,6 a 3,5. La total incidencia de cáncer ha disminuido debido al decrecimiento de cáncer de células escamosas, células grandes y pequeñas y se extiende a todos los grupos. La incidencia de adenocarcinomas se estabilizó, creció desde 1988-1990 y después se mantuvo estable. El inicial incremento se centró en los grupos de más edad. Estos patrones temporales sugieren que los mismos factores etiológicos afectan a ambos géneros; habría que hacer más estudios para cuantificar estos factores no debidos al tabaco.

De acuerdo con la Encuesta Nacional de Salud $2001^{28}$, la prevalencia del tabaquismo en España, es decir el porcentaje de personas que declaran haber fumado en los últimos 30 días, en población de 16 años y más, se sitúa en el 34,4\% (el 31,7\% lo hace diariamente). Por sexos, destaca que los hombres (42\%) en conjunto todavía fuman más que las mujeres $(27 \%)$. La tendencia en aquéllos sigue siendo descendente, mientras que en las mujeres es ascendente. Por grupos de edad, destaca que las chicas de entre 16 y 24 años fuman más que los chicos $(42,7 \%$ frente al $40,8 \%$ ). Entre los que fuman a diario, el promedio de cigarrillos consumidos es de 17.

En la Comunidad Autónoma de Aragón hay una prevalencia de consumo diario menor a la media nacional (29,4\% frente al 31,7\%), tanto en total como para ambos sexos. Entre los adolescentes, según los resultados de la Encuesta sobre Drogas a la Población Escolar 2002, se desprende que el porcentaje de estudiantes de 14 a 18 años que declara haber fumado alguna vez se sitúa en el $69,5 \%$. En la actualidad fuman el $34,5 \%$, siendo la prevalencia mayor entre las chicas $(36,6 \%)$ que entre los chicos $(32 \%)$.

Estas cifras, todavía alarmantes en torno al consumo de tabaco, están en correspondencia con los resultados vistos de cáncer de pulmón y la esperanza no es buena si no se restringen. Sin embargo no hay que desatender a otros factores de riesgo presentes en ambos sexos relacionados con la contaminación ambiental ${ }^{29}$ y patrones genéticos ${ }^{30} \mathrm{y}$ de la susceptibilidad especial que según algunos estudios asocian la mayor susceptibilidad del tabaco a cáncer de pulmón en mujeres. Cualquiera de los factores de ries- go conocidos o esperados deben de tenerse en cuenta en este incremento observado de cáncer de pulmón en mujeres y tratar de controlar el tabaquismo al igual que otros factores de riesgo.

El perfil de la mujer con cáncer de pulmón en nuestro país sería por tanto: mujer de 64 años, fumadora que debuta en estadios avanzados, con un $1 \%$ de supervivencia a los 5 años en estadio IV. Por tanto se hace más necesario que nunca insistir en el control del tabaco y otros posibles agentes cancerigenos con el fin de disminuir esta enfermedad en nuestra colectividad.

\author{
Correspondencia: \\ Dr. M. Bernal \\ Comité de Tumores del Tórax \\ Hospital Clínico Universitario Lozano Blesa \\ Facultad de Medicina \\ Domingo Miral, s/n \\ E-50009 Zaragoza \\ E-mail: mibernal@unizar.es
}

\section{Bibliografía}

1. Bernal M. Incremento del cáncer de pulmón en mujeres. Med Clin (Barc) 2002;119(11):435-6.

2. Mountain CF. A new international staging system for lung cancer. Chest 1997;111(6):1711-17.

3. Hernández Hernández JR, Tapias del Pozo JA, Moreno Canelo P, Rodríguez Puebla A, Paniagua Tejo S, Sánchez Marcos JC. Incidencia del cáncer de pulmón en la provincia de Ávila en 2002. Arch Bronconeumol 2004;40(7):304-10.

4. Borras J, Borras JM, Galcerán J, Sánchez V, Moreno V, González JR. Tendencia del cáncer relacionado con el tabaco en Tarragona, España, 1980-96. Cancer Causes Control 2001:12(10):903-8.

5. Registro de Cáncer de Navarra, Sección de Enferedades no Transmisibles y Estadísticas Vitales. Incidence and mortality of cancer in Navarra, 1993-1997. Tendencies in the last 25 years. An Sist Sanit Navar 2001;24(3):339-62.

6. Rodríguez Suárez V, Álvarez Sánchez G, Díaz Ruisánchez E, García Tardón A. Tendencia de la mortalidad por cáncer de pulmón en Asturias (1976-1989). Rev San Hig Pub 1993;67:465-474.

7. Crocetti E, Capocaccia R, Casella C, Guzzinati S, Ferretti S, Rosso S, Sacchettini C, Spitale A, Stracci F, Tumino R. Network of the Italian Cancer Registries (AIRT). Population-based incidence and mortality cancer trends (19861997) from the network of Italian cancer registries. Eur J Cancer Prev 2004;13(4):287-95. 
8. Crocetti E, Paci E, Miccinesi G, Costantini AS, Zappa M. Time trends in cancer incidence and mortality in central Italy. Eur J Cancer Prev 2002;11(4):387-95.

9. Crocetti E, Capocaccia R, Casella C, Ferretti S, Guzzinati S, Rosso S, Sacchettini C, Spitale A, Stracci F, Tumino R. Cancer trends in Italy: figures from the cancer registries (1986-1997). Epidemiol Prev 2004;28(2 Suppl):1-6.

10. Siesling S, van Dijck JA, Visser O, Coebergh JW. Working Group of The Netherlands Cancer Registry. Trends in incidence of and mortality from cancer in The Netherlands in the period 1989-1998. Eur J Cancer 2003;39(17):252130.

11. Remontet L, Esteve J, Bouvier AM, Grosclaude P, Launoy G, Menegoz F, Exbrayat C, Tretare B, Carli PM, Guizard AV, Troussard X, Bercelli P, Colonna M, Halna JM, Hedelin G, Mace-Lesec'h J, Peng J, Buemi A, Velten M, Jougla E, Arveux P, Le Bodic L, Michel E, Sauvage M, Schvartz C, Faivre J. Cancer incidence and mortality in France over the period 1978-2000. Cancer incidence and mortality in France over the period 1978-2000. Rev Epidemiol Sante Publique 2003;51(1 Pt 1):3-30.

12. Swerdlow AJ, dos Santos Silva I, Reid A, Qiao Z, Brewster $\mathrm{DH}$, Arrundale J. Trends in cancer incidence and mortality in Scotland: description and possible explanations. Br J Cancer 1998;77 Suppl 3:1-54.

13. Peto R, Darby S, Deo H, Silcocks P, Whitley E, Doll R. Smoking, smoking cessation, and lung cancer in the UK since 1950: combination of national statistics with two case-control studies. BMJ 2000;321(7257):323-9.

14. Hakulinen T, Andersen A, Malker B, Pukkala E, Schou G, Tulinius H. Trends in cancer incidence in the Nordic countries. A collaborative study of the five Nordic Cancer Registries. Acta Pathol Microbiol Immunol Scand Suppl 1986;288:1-151.

15. Bray F, Tyczynski JE, Parkin DM. Going up or coming down? The changing phases of the lung cancer epidemic from 1967 to 1999 in the 15 European Union countries. Eur J Cancer 2004;40(1):96-125.

16. Devesa SS, Blot WJ, Stone BJ, Miller BA, Tarone RE, Fraumeni JF Jr. Recent cancer trends in the United States. J Natl Cancer Inst 1995 1;87(3):159-60.

17. Islam SS, Schottenfeld D.Declining FEV1 and chronic productive cough in cigarette smokers: a 25 -year prospective study of lung cancer incidence in Tecumseh, Michigan. Cancer Epidemiol Biomarkers Prev 1994 Jun;3(4):289-98.

18. Merrill RM. Measuring the projected public health impact of lung cancer through lifetime and age-conditional risk estimates. Ann Epidemiol 2000;10(2):88-96.
19. Trapido EJ, Burciaga Valdez R, Obeso JL, Strickman-Stein N, Rotger A, Perez-Stable EJ. Epidemiology of cancer among Hispanics in the United States. J Natl Cancer Inst Monogr 1995;(18):17-28.

20. Toh CK, Wong EH, Lim WT, Leong SS, Fong KW, Wee J, Tan EHThe Impact of Smoking Status on the Behavior and Survival Outcome of Patients With Advanced Non-small Cell Lung Cancer: A Retrospective Analysis. Chest 2004 Dec;126(6):1750-6.

21. Thongprasert S, Chewaskulyong B. Study of cisplatin-vinblastine plus amifostine in patients with locally advanced or metastatic non-small cell lung cancer. J Med Assoc Thai $2004 ; 87(10): 1162-7$.

22. Grivaux M, Breton JL, Bombaron P, Kuntz P, Lebas FX, Mehdaoui A, Herman D, David P, Berruchon J, Delclaux B, Zureik M, Blanchon F. Lung cancer among women in France. Analysis of the 904 French women with lung cancer. Lung Cancer 2004;45(3):279-87.

23. Radzikowska E, Glaz P, Roszkowski K. Lung cancer in women: age, smoking, histology, performance status, stage, initial treatment and survival. Population-based study of 20561 cases. Ann Oncol 2002;13(7):1087-93.

24. Piquet J, Blanchon F, Grivaux M, Collon T, Zureik M, Barbieux H, Benichou-Flurin M, Breton JL, Coetmeur D, Delclaux B, Braun D, Homasson JP, Mouysset F, Riou R, le Poulain-Doubliez M, Marsal L. PCG. Primary bronchial carcinoma in elderly subjects in France. Rev Mal Respir 2003;20(5 Pt 1):691-9.

25. Ambrosone CB, Rao U, Michalek AM, Cummings KM, Mettlin CJ. Lung cancer histologic types and family history of cancer. Analysis of histologic subtypes of 872 patients with primary lung cancer. Cancer 1993 15;72(4):1192-8.

26. Charloux A, Rossignol M, Purohit A, Small D, Wolkove N, Pauli G, Quoix E, Kreisman H. International differences in epidemiology of lung adenocarcinoma. Lung Cancer 1997;16(2-3):133-43.

27. Chiu YL,Yu IT,Wong TW. Time trends of female lung cancer in Hong Kong: Age,period and analysis. Int J Cancer 2004;111:424-30.

28. Instituto de Información Sanitaria. Encuesta Nacional de salud Año 2001. Ministerio de Sanidad y Consumo. Madrid 2003.

29. Wichmann HE, Rosario AS, Heid IM, Kreuzer M, Heinrich J, Kreienbrock L. Increased lung cancer risk due to residential radon in a pooled and extended analysis of studies in Germany. Health Phys 2005;88(1):71-9.

30. Blot WJ, McLaughlin JK. Are women more susceptible to lung cancer? JNCI 2004;96:812-813. 